\title{
New high yielding and disease resistant mungbean [Vigna radiata (L.) Wilczek] variety "NIFA Mung-19"
}

Gul Sanat Shah Khattak, Iqbal Saeed* and Muhammad Ibrar

Plant Breeding \& Genetics Division, Nuclear Institute for Food \& Agriculture (NIFA), Peshawar, Khyber

Pakhtunkhwa-Pakistan

*Corresponding author's email: iqbal.saeed@yahoo.com

Citation

Gul Sanat Shah Khattak, Iqbal Saeed and Muhammad Ibrar. New high yielding and disease resistant mungbean [Vigna radiata (L.) Wilczek] variety "NIFA Mung-19". Pure and Applied Biology. Vol. 8, Issue 2, pp1444-1455. http://dx.doi.org/10.19045/bspab.2019.80084

Received: $25 / 01 / 2019$

Revised: $24 / 04 / 2019$

Accepted: 30/04/2019

Online First: 09/05/2019

\section{Abstract}

Mungbean is an important kharif crop of Pakistan as well as the Khyber Pakhtunkhwa (KP) province. The overall yield of this crop is low and one of the main reasons is the poor genetic background of the existing mungbean varieties in the country. Mungbean varieties adapted to the growing conditions have purple hypocotyls, whereas mungbean varieties with green hypocotyls have an additional potential for sprouts production. In order to replenish the existing pool of mungbean varieties with better ones, a locally adapted mungbean variety "NM 92" and an exotic Mungbean Yellow Mosaic Virus (MYMV) susceptible variety "VC 1560D" were crossed (NM 92 x VC 1560D) in kharif 2004 at Nuclear Institute for Food and Agriculture (NIFA), Peshawar. Single plants selections for more pods plant ${ }^{-1}$, semi erect growth, purple/green hypocotyls color, resistance to MYMV disease, and confirmation of breeding behavior of these traits were carried-out in $\mathrm{F}_{2}-\mathrm{F}_{5}$ generations during 2006-2010. One of the recombinants designated as NFM-5-36-27 was selected on the basis of overall better performance with green hypocotyls color, and further evaluated in disease screening nurseries and various replicated yield trials. NFM-5-36-27 produced seed yield of 530 to $2076 \mathrm{~kg} \mathrm{ha}^{-1}$ in replicated yield trials conducted during different years and locations compared with the check variety Ramzan $\left(316-1417 \mathrm{~kg} \mathrm{ha}^{-1}\right)$ with $38 \%$ to $68 \%$ increases in seed yield over Ramzan. In Mungbean National Uniform Yield Trial conducted in 2015 and 2017, NFM-5-36-27 produced higher seed yields of 725 and $996 \mathrm{~kg} \mathrm{ha}^{-1}$, respectively compared with national checks. NFM-536-27 has a future potential for mungbean sprouts production in the country due to its green hypocotyls color. NFM-5-36-27 was recommended as new mungbean commercial variety with the name "NIFA Mung -19 by the KP Provincial Seed Council in its $38^{\text {th }}$ meeting held on $3^{\text {rd }}$ January, 2018 at Peshawar.

Keywords: disease; high yield; hypocotyls; recombinant; variety; Vigna radiata

\section{Introduction}

Among kharif season pulses, mungbean (Vigna radiata (L.) Wilczek) enjoys a unique position as it is grown on marginal lands of the country with negligible external inputs, therefore other major kharif crops e.g. maize, cotton, rice etc cannot find place in such cropping system because of requirements for huge quantities of external inputs. Currently, mungbean is grown on an area of 178.7 thousand hectares in Pakistan and 7.5 thousand hectares in KP [1]. The average national yield of mungbean is far below as compared to other neighboring countries. This is mainly because of the limited availability of local mungbean varieties with 
high yield potential as well as strong base for disease resistance, which in turn is the result of narrow genetic base of most of the local varieties. As pulses are grown by the farmers with small land-holdings, the only attractive trait of a new commercial variety is the higher grain yield [2,3], and easy availability of such varieties to the farmers ensures harvesting of benefits from these varieties [4, 5]. New seed varieties ensure yield enhancement as well as higher per unit land production as compared to the traditional crop varieties [6]. It is therefore possible to increase both production and area under mungbean cultivation in the $\mathrm{KP}$ and the country as well through development of improved mungbean varieties.In order to fill the gap, agricultural research institutes in the country are taking efforts to develop new high yielding mungbean varieties with major share being contributed by the Punjab Province, and have released few commercial varieties with better yield potential and disease resistance [7]. These varieties perform well under local environmental conditions, whereas their yield performance does not remain stable under KP's growth conditions possibly due to differences in environmental conditions. Nuclear Institute for Food and Agriculture (NIFA), Peshawar realized this need and initiated an organized Research \& Development (R\&D) work on mungbean, and developed/evolved two high yielding and disease resistant varieties namely "Ramzan" [8] and NIFA Mung - 17 best suited to the environmental conditions of the KP.

Mungbean Yellow Mosaic Virus (MYMV) disease is devastating to overall mungbean production, and the only viable solution is the development of mungbean varieties with inherent potential to withstand this disease with little or no impact on seed yield. MYMV disease resistance is the effect of certain modifying genes [9], and gathering such favorable genes in a single high yielding genotype guarantees protection of seed yield potential of that particular genotype/cultivar. It further improves overall acceptability and adoptability of such varieties among mungbean growers.

Apart from other uses, mungbean is also valued for sprouts production, and these are an important part of daily diets in many countries e.g. China, Malaysia, Korea etc [10]. Mungbean sprouts are only produced from mungbean plant types having green hypocotyls color. If proper markets for mungbean sprouts are explored in Pakistan, this will positively enhance revenue generated from mungbean crop, and will contribute towards uplifting living standards of farmers of marginal lands in the country. The current manuscript discusses development/evolution of new high yielding mungbean variety "NIFA Mung - 19 .

\section{Materials and Methods}

An exotic mungbean accession "VC1560D" susceptible to MYMV disease from the Asian Regional Centre - Asian Vegetable Research and Development Centre (ARC - AVRDC), Thailand, and a local MYMV resistant cultivar "NM 92" were used in the current study. These were hybridized as single crosscombination (NM $92 \times$ VC 1560D) following an efficient mungbean crossing technique [11] at the research farm of the Nuclear Institute for Food and Agriculture (NIFA), Peshawar during kharif, 2004. $F_{1}$ generation from this cross-combination was planted at NIFA, Peshawar during summer 2005. All recombinant plants were harvested individually and planted as $F_{2}$ population along with both parents for comparison during kharif 2006. The MYMV susceptible parent "VC 1560D" was planted as repeated rows through the entire segregating population. Single plants bearing more pods, more productive branches, MYMV resistance, semi-spreading growth habit, and green/purple hypocotyls color were selected. The selected recombinant single plants were 
planted as plant-progeny-rows in kharif 2007 and further single plants selections based on the above-mentioned criteria was undertaken. The selected recombinants were further planted in $\mathrm{F}_{4}-\mathrm{F}_{5}$ generations for generation advancement and confirmation of breeding behavior and genetic stability of the target traits during kharif 2008 and 2010, and better performing single lines were subsequently selected.

The selected single lines were evaluated for yield potential in various replicated yield trials i.e. Preliminary Yield Trails (PYTs), Advanced Lines Yield Trials (ALYTs) at NIFA, Peshawar and Adaptation Yield Trials (AYTs) at other locations outside NIFA during 2011 - 2017. Based on performance in these trials, the recombinant designated as NFM-5-36-27 was finally selected and tested for wider adaptability and yield stability across country in Mungbean National Uniform Yield Trial (NUYT) during 2015 and 2017. The experimental material in all replicated trials was laid-out in Randomized Complete Block Design (RCBD) with each plot comprising of 04 rows of 04 meter length. Plant-to-plant and row-to-row distances were maintained at $10 \mathrm{~cm}$ and 30 , respectively, and each plot was replicated three times. The crop was sown under irrigated conditions and irrigation was applied as and when required. All statistical analyses were performed according to Steel and Torrie, [12]. MYMV disease reaction data was recorded following standard procedure [13]. Breeding history of NIFA Mung - 19 (recombinant NFM-5-3627 ) is presented in (Table 1).

\section{Table 1. Breeding history of NIFA Mung-19 (recombinant NFM-5-36-27)}

\begin{tabular}{|c|c|c|c|}
\hline S. No. & Year & F. Generation/Trial & Remarks \\
\hline 1 & $\begin{array}{l}\text { Kharif } \\
2004\end{array}$ & $\mathrm{~F}_{0}$ & $\begin{array}{l}\text { A single cross "VC 1560D x NM 92" was attempted } \\
\text { between an exotic accession "VC1560D" and a local } \\
\text { commercial variety "NM92" at Nuclear Institute for Food } \\
\text { and Agriculture (NIFA), Peshawar. All crossed pods were } \\
\text { picked. }\end{array}$ \\
\hline 2 & $\begin{array}{l}\text { Summer } \\
2005\end{array}$ & $\mathrm{~F}_{1}$ & $\begin{array}{l}\mathrm{F}_{1} \text { generation of the cross "VC } 1560 \mathrm{D} \times \mathrm{NM} 92 \text { " was } \\
\text { raised. All hybrid plants were harvested, threshed and } \\
\text { bagged individually. }\end{array}$ \\
\hline 3 & $\begin{array}{l}\text { Kharif } \\
2006\end{array}$ & $\mathrm{~F}_{2}$ & $\begin{array}{l}\mathrm{F}_{2} \text { populations were raised as plant-progeny-rows from } \\
\text { plants harvested from } \mathrm{F}_{1} \text { generation along with parents. } \\
\text { Populations were also screened for MYMV. Selections } \\
\text { were made on the basis of both green/purple hypocotyls, } \\
\text { more number of pods, resistance to MYMV and semi } \\
\text { spreading plant type }\end{array}$ \\
\hline 4 & $\begin{array}{l}\text { Kharif } \\
2007\end{array}$ & $\mathrm{~F}_{3}$ & $\begin{array}{l}\text { Plant populations along with parents were planted as } \mathrm{F}_{3} \\
\text { generation for confirmation of breeding behavior and } \\
\text { generation advancement. Populations were also screened } \\
\text { for MYMV. Single plants selections were made for } \\
\text { green/purple hypocotyls color, more pods, resistance to } \\
\text { MYMV and good plant type, based on visual } \\
\text { observations. }\end{array}$ \\
\hline 5 & $\begin{array}{l}\text { Kharif } \\
2008-10\end{array}$ & $\begin{array}{c}\mathrm{F}_{4} / \mathrm{F}_{5} \text {, and screening } \\
\text { against MYMV }\end{array}$ & $\begin{array}{l}\text { Selected plants were planted as plant-progeny-rows in } \mathrm{F}_{4} \\
\text { generation along with parents for generation advancement } \\
\text { and to confirm breeding behavior of the traits. Screening } \\
\text { was also carried-out for MYMV behavior. True breeding } \\
\text { lines with desired hypocotyls color, more pods, good plant } \\
\text { stand and resistance to MYMV were selected to confirm } \\
\text { their breeding behavior. The selected lines were advanced }\end{array}$ \\
\hline
\end{tabular}




\begin{tabular}{|c|c|c|c|}
\hline & & & $\begin{array}{l}\text { as line-progeny-rows for seed increase, and confirmation } \\
\text { of breeding behavior and MYMV resistance. }\end{array}$ \\
\hline 6 & $\begin{array}{c}\text { Kharif } \\
2011-17\end{array}$ & $\begin{array}{c}\text { Yield Trials and } \\
\text { screening against } \\
\text { MYMV }\end{array}$ & $\begin{array}{c}\text { The recombinant lines selected for desired hypocotyls } \\
\text { color, high yield and MYMV resistance were further } \\
\text { evaluated for agronomic traits in replicated preliminary } \\
\text { yield trials and also screened for MYMV in a separate } \\
\text { nursery at NIFA, Peshawar. } \\
\text { The advanced recombinant lines selected from } \\
\text { preliminary yield trials on the basis of high yield potential, } \\
\text { good plant type and resistance to MYMV were further } \\
\text { evaluated in advanced lines and adaptation yield trials. } \\
\text { The better performing recombinant line "NFM-5-36-27" } \\
\text { with green hypocotyls color was evaluated for } \\
\text { Distinctness, Uniformity and Stability (DUS) studies and } \\
\text { National Uniform Yield trials. }\end{array}$ \\
\hline
\end{tabular}

\section{Results and discussion}

Results of yield and yield components for NFM-5-36-27 (NIFA Mung - 19) are shown in (Table 2 to 8). In Preliminary Yield Trials (PYTs) conducted at NIFA, Peshawar during kharif 2011 and 2012, significant and higher seed yields of 1579 and $1892 \mathrm{~kg} \mathrm{ha}^{-1}$ were produced by NFM-5-36-27 compared with check variety Ramzan (1314 and $1417 \mathrm{~kg} \mathrm{ha}^{-1}$, respectively (Table $2 \& 3$ ). NFM-5-36-27 also produced significant and higher seed yields of 2076 and $1906 \mathrm{~kg} \mathrm{ha}^{-1}$ against seed yields of 1386 and $1189 \mathrm{~kg} \mathrm{ha}^{-1}$ produced by check variety Ramzan in Advanced Lines Yield Trials (ALYTs) conducted at NIFA, Peshawar during Kharif 2014 and 2015, respectively (Table 4 \& 5). Similarly, in Adaptation Yield Trials (AYTs) conducted at Agricultural Research Station (ARS), Karak during 2016 and 2017, NFM-5-36-27 out yielded check variety Ramzan and produced significant and higher averaged seed yield of $530 \mathrm{~kg} \mathrm{ha}^{-1}$ as compared to averaged seed yield of $316 \mathrm{~kg}$ ha ${ }^{1}$ produced by the check variety with $68 \%$ increase in seed yield over check variety (Table 6). NFM-5-36-27 also produced significant and higher seed yield of $983 \mathrm{~kg} \mathrm{ha}^{-1}$ at Arid Zone Research Centre (AZRC), Dera Ismail Khan (D. I. Khan) during kharif 2017, whereas check variety Ramzan produced seed yield of $599 \mathrm{~kg}$ ha $^{-1}$ (Table 7). In Adaptation Yield Trial (AYT) conducted on farmer's field at Kuram during Kharif 2016 and 2017, NFM-5-36-27 produced significant and higher averaged seed yield of $1855 \mathrm{~kg} \mathrm{ha}^{-1}$ compared with check variety Ramzan (averaged seed yield of $1347 \mathrm{~kg} \mathrm{ha}^{-1}$ ) with $38 \%$ increase in seed yield over check variety (Table 8). NIFA Mung-19 (code name NIFA MUNG-5) also performed well compared with the national check varieties in National Uniform Yield Trials (NUYTs) conducted by the National Pulses Coordinator, National Agricultural Research Centre (NARC), Islamabad in 2015 and 2017. NIFA Mung-19 produced higher averaged seed yield of $725 \mathrm{~kg} \mathrm{ha}^{-1}$ compared with AZRI Mung-06 $\left(700 \mathrm{~kg} \mathrm{ha}^{-1}\right), \mathrm{NM}-2011\left(634 \mathrm{~kg} \mathrm{ha}^{-1}\right)$ and NM$06\left(649 \mathrm{kh} \mathrm{ha}^{-1}\right)$ in the NUYT conducted in 2015 (Table 9). Similarly, NIFA Mung-19 (code name NIFA MUNG-5) also produced high averaged seed yield of $996 \mathrm{~kg} \mathrm{ha}^{-1}$ compared with averaged seed yield of $975 \mathrm{~kg}$ $\mathrm{ha}^{-1}$ and $919 \mathrm{~kg} \mathrm{ha}^{-1}$ produced by NM-2011 and AZRI Mung-06, respectively in the NUYT conducted in 2017 (Table 10). High yielding varieties of crop plants help in increasing yield as well as production [14-17] and high grain yield potential is the only trait that is highly regarded by the growers $[2,3]$. The high yield potential of NIFA Mung-19 (Table 2 to 8) will help a great deal in alleviating constraints to improve mungbean production in the $\mathrm{KP}$ in particular and the country in general. One of the pre-requisite for new crop variety is the new variety must surpass the existing ones in terms of seed yield and other agronomic traits as well 
as adaptability across locations. $65 \%$ and $38 \%$ increase in seed yield by NIFA Mung - 19 over check variety in Adaptation Yield Trials (Table
$7 \& 8$ ) hints about its high yield potential and wider adaptability in the Province and in the country as well.

Table 2. Performance of NFM-5-36-27 (NIFA Mung-19) in preliminary yield trial conducted during kharif 2011 at NIFA, Peshawar

\begin{tabular}{|c|c|c|c|c|c|c|}
\hline Entry & Parentage & $\begin{array}{c}\text { Days to } \\
\text { flowering }\end{array}$ & $\begin{array}{c}\text { Days to } \\
\text { maturity }\end{array}$ & $\begin{array}{c}\text { Plant } \\
\text { ht. (cm) }\end{array}$ & $\begin{array}{c}\text { 1000 seed } \\
\text { weight (g) }\end{array}$ & $\begin{array}{c}\text { Yield } \\
\text { kg/ha }\end{array}$ \\
\hline NFM-5-36-27 & VC1560D x NM92 & 48 & 69 & 50 & 54 & 1579 \\
\hline NFM-5-36-29 & -do- & 46 & 67 & 46 & 58 & 1368 \\
\hline NFM-5-36-30 & -do- & 43 & 71 & 48 & 53 & 1212 \\
\hline NFM-5-36-33 & -do- & 41 & 71 & 43 & 51 & 710 \\
\hline NFM-5-36-41 & -do- & 42 & 71 & 53 & 55 & 1373 \\
\hline NFM-5-36-42 & -do- & 44 & 69 & 62 & 42 & 608 \\
\hline NFM-5-36-43 & -do- & 47 & 72 & 56 & 55 & 1368 \\
\hline NFM-5-36-45 & -do- & 44 & 70 & 44 & 53 & 1458 \\
\hline NFM-5-36-48 & -do- & 44 & 69 & 39 & 65 & 1056 \\
\hline NFM-5-36-49 & -do- & 44 & 71 & 39 & 53 & 912 \\
\hline NFM-5-36-50 & -do- & 43 & 69 & 46 & 65 & 1167 \\
\hline NFM-5-36-51 & -do- & 42 & 71 & 39 & 51 & 1370 \\
\hline NFM-5-36-52 & -do- & 44 & 74 & 40 & 56 & 1161 \\
\hline NFM-5-36-53 & -do- & 43 & 67 & 44 & 61 & 1247 \\
\hline NFM-5-36-55 & -do- & 45 & 69 & 51 & 64 & 1689 \\
\hline Ramzan (Check) & VC 1482C x NM 92 & 44 & 68 & 49 & 52 & 1314 \\
\hline CV & & 0.56 & 0.80 & 0.73 & 0.52 & 24.90 \\
\hline LSD 5\% & & 1.15 & 1.64 & 1.49 & 1.06 & 50.84 \\
\hline
\end{tabular}

Table 3. Performance of NFM-5-36-27 (NIFA Mung-19) in preliminary yield trial conducted during kharif 2012 at NIFA, Peshawar

\begin{tabular}{|c|c|c|c|c|c|c|}
\hline Entry & Parentage & $\begin{array}{c}\text { Days to } \\
\text { flowering }\end{array}$ & $\begin{array}{c}\text { Days to } \\
\text { maturity }\end{array}$ & $\begin{array}{c}\text { Plant ht. } \\
(\mathbf{c m})\end{array}$ & $\begin{array}{c}\mathbf{1 0 0 0} \text { seed } \\
\text { wt. (g) }\end{array}$ & $\begin{array}{c}\text { Yield } \\
\text { kg/ha }\end{array}$ \\
\hline NFM-5-36-27 & VC1560D x NM92 & 44 & 73 & 71 & 53 & 1892 \\
\hline NFM-5-36-29 & -do- & 41 & 74 & 78 & 50 & 1403 \\
\hline NFM-5-36-30 & -do- & 43 & 76 & 68 & 51 & 1597 \\
\hline NFM-5-36-33 & -do- & 41 & 74 & 68 & 47 & 1444 \\
\hline NFM-5-36-41 & -do- & 38 & 74 & 67 & 51 & 1448 \\
\hline NFM-5-36-42 & -do- & 43 & 74 & 63 & 46 & 1340 \\
\hline NFM-5-36-43 & -do- & 42 & 74 & 68 & 54 & 1472 \\
\hline NFM-5-36-45 & -do- & 40 & 74 & 75 & 48 & 1306 \\
\hline NFM-5-36-48 & -do- & 40 & 74 & 71 & 58 & 1747 \\
\hline NFM-5-36-49 & -do- & 40 & 74 & 78 & 50 & 1604 \\
\hline NFM-5-36-50 & -do- & 43 & 75 & 65 & 60 & 1896 \\
\hline NFM-5-36-51 & -do- & 40 & 75 & 71 & 50 & 1556 \\
\hline NFM-5-36-52 & -do- & 39 & 76 & 75 & 54 & 1663 \\
\hline NFM-5-36-53 & -do- & 42 & 74 & 74 & 56 & 1590 \\
\hline NFM-5-36-55 & -do- & 42 & 76 & 68 & 59 & 1684 \\
\hline Ramzan (Check) & VC 1482C x NM 92 & 39 & 74 & 69 & 50 & 1417 \\
\hline CV & & 1.33 & 0.88 & 3.02 & 0.22 & 69.91 \\
\hline LSD 5\% level & & 3.85 & 2.55 & 8.73 & 0.32 & 201.90 \\
\hline
\end{tabular}


Table 4. Performance of NFM-5-36-27 (NIFA Mung-19) in advanced lines yield trial conducted duringkharif 2014 at NIFA, Peshawar

\begin{tabular}{|c|c|c|c|c|c|c|}
\hline Entry & Parentage & $\begin{array}{c}\text { Days to } \\
\text { flowering }\end{array}$ & $\begin{array}{c}\text { Days to } \\
\text { maturity }\end{array}$ & $\begin{array}{c}\text { Plant ht. } \\
(\mathbf{c m})\end{array}$ & $\begin{array}{c}\text { 1000 seed } \\
\text { wt. (g) }\end{array}$ & $\begin{array}{c}\text { Yield } \\
\text { kg/ha }\end{array}$ \\
\hline NFM-5-36-27 & VC1560D x NM92 & 33 & 94 & 90 & 51 & 2076 \\
\hline NFM-5-36-29 & -do- & 32 & 93 & 77 & 52 & 2069 \\
\hline NFM-5-36-30 & -do- & 32 & 92 & 87 & 45 & 2115 \\
\hline NFM-5-36-33 & -do- & 33 & 94 & 77 & 46 & 1795 \\
\hline NFM-5-36-41 & -do- & 35 & 94 & 84 & 48 & 1823 \\
\hline NFM-5-36-42 & -do- & 36 & 94 & 94 & 45 & 1215 \\
\hline NFM-5-36-43 & -do- & 36 & 93 & 88 & 44 & 997 \\
\hline NFM-5-36-45 & -do- & 36 & 92 & 97 & 54 & 2021 \\
\hline NFM-5-36-48 & -do- & 36 & 92 & 88 & 54 & 1976 \\
\hline NFM-5-36-49 & -do- & 37 & 92 & 88 & 50 & 2056 \\
\hline NFM-5-36-50 & -do- & 37 & 91 & 90 & 51 & 1750 \\
\hline NFM-5-36-51 & -do- & 37 & 91 & 90 & 44 & 1813 \\
\hline NFM-5-36-52 & -do- & 37 & 92 & 85 & 51 & 1403 \\
\hline NFM-5-36-53 & -do- & 37 & 92 & 66 & 53 & 2063 \\
\hline NFM-5-36-55 & -do- & 37 & 91 & 78 & 52 & 2236 \\
\hline Ramzan (Check) & VC 1482C x NM & 37 & 90 & 66 & 48 & 1396 \\
\hline 92 & & 2.3 & 2.7 & 2.1 & 1.4 & 13.2 \\
\hline LSD 5\% level & & 3.5 & 3.5 & 5.3 & 2.1 & 187 \\
\hline
\end{tabular}

Table 5. Performance of NFM-5-36-27 (NIFA Mung-19) in advanced lines yield trial conducted during kharif 2015 at NIFA, Peshawar

\begin{tabular}{|c|c|c|c|c|c|c|}
\hline Entry & Parentage & $\begin{array}{c}\text { Days to } \\
\text { flowering }\end{array}$ & $\begin{array}{c}\text { Days to } \\
\text { maturity }\end{array}$ & $\begin{array}{c}\text { Plant } \\
\text { ht. (cm) }\end{array}$ & $\begin{array}{c}\text { 1000 seed } \\
\text { wt. (g) }\end{array}$ & $\begin{array}{c}\text { Yield } \\
\text { kg/ha }\end{array}$ \\
\hline NFM-5-36-1 & VC 1560D x NM 92 & 46 & 84 & 89 & 46 & 1902 \\
\hline NFM-5-36-2 & -do- & 50 & 91 & 93 & 50 & 1523 \\
\hline NFM-5-36-4 & -do- & 47 & 87 & 91 & 51 & 1475 \\
\hline NFM-5-36-11 & -do- & 49 & 91 & 92 & 55 & 1549 \\
\hline NFM-5-36-24 & -do- & 46 & 82 & 92 & 53 & 1731 \\
\hline NFM-5-36-27 & -do- & 48 & 89 & 84 & 53 & 1906 \\
\hline NFM-5-63-8 & VC1482C x NM92 & 52 & 90 & 89 & 48 & 1277 \\
\hline NFM-5-63-10 & -do- & 48 & 91 & 90 & 56 & 1115 \\
\hline NFM-5-63-14 & -do- & 50 & 90 & 90 & 49 & 1322 \\
\hline NFM-5-63-30 & -do- & 44 & 76 & 90 & 53 & 1718 \\
\hline NFM-5-63-35 & -do- & 47 & 91 & 92 & 53 & 1342 \\
\hline NFM-5-63-43 & -do- & 47 & 91 & 92 & 57 & 1316 \\
\hline NFM-5-63-48 & -do- & 44 & 78 & 90 & 55 & 1539 \\
\hline NFM-5-63-49 & -do- & 46 & 91 & 89 & 50 & 1536 \\
\hline NFM-5-63-51 & -do- & 45 & 81 & 90 & 46 & 1789 \\
\hline Ramzan (Check) & VC 1482C x NM 92 & 49 & 85 & 79 & 51 & 1189 \\
\hline CV & & 2.3 & 2.4 & 4.5 & 2.6 & 6.1 \\
\hline LSD 5\% level & & 3.5 & 5.4 & 4.9 & 4.3 & 155 \\
\hline
\end{tabular}


Table 6. Performance of NFM-5-36-27 (NIFA Mung-19) in mungbean adaptation yield trials planted at ARS, Karak during 2016 and 2017

\begin{tabular}{|c|c|c|c|c|c|c|c|c|}
\hline \multirow{2}{*}{ Entry } & \multirow{2}{*}{ Parentage } & \multirow{2}{*}{$\begin{array}{c}\text { Days to } \\
\text { flowering }\end{array}$} & \multirow{2}{*}{$\begin{array}{l}\text { Days to } \\
\text { maturity }\end{array}$} & \multirow{2}{*}{$\begin{array}{l}\text { Plant ht. } \\
\quad(\mathrm{cm})\end{array}$} & \multicolumn{3}{|c|}{ Yield (kg ha-1) } & \multirow{2}{*}{$\begin{array}{c}\% \text { increase in seed yield } \\
\text { over check }\end{array}$} \\
\hline & & & & & 2016 & 2017 & Average & \\
\hline NFM-8-22 & NM93 x NM92 & 53 & 74 & 45 & 577 & 257 & 417 & 32 \\
\hline NFM-5-36-1 & VC1560D x NM92 & 52 & 74 & 37 & 687 & 194 & 441 & 40 \\
\hline NFM-5-36-27 & VC1560D x NM92 & 54 & 74 & 41 & 726 & 333 & 530 & 68 \\
\hline NFM-5-63-30 & VC1482C x NM92 & 53 & 71 & 40 & 549 & 298 & 424 & 35 \\
\hline NFM-5-91-26 & NM98 x VC3902A & 54 & 71 & 39 & 543 & 201 & 372 & 18 \\
\hline Ramzan (Check) & VC $1482 C$ x NM 92 & 54 & 70 & 37 & 445 & 187 & 316 & - \\
\hline $\mathrm{CV}$ & & 1.04 & 0.81 & 1.32 & 2.19 & 2.29 & & \\
\hline LSD (5\%) & & 0.94 & 1.0 & 1.23 & 20.1 & 15.35 & & \\
\hline
\end{tabular}

Table 7. Performance of NFM-5-36-27 (NIFA Mung-19) in mungbean adaptation yield trial planted at AZRC, D.I. Khan during 2017

\begin{tabular}{|c|c|c|c|c|c|c|}
\hline Entry & Parentage & $\begin{array}{c}\text { Days to } \\
\text { flowering }\end{array}$ & $\begin{array}{c}\text { Days to } \\
\text { maturity }\end{array}$ & $\begin{array}{c}\text { Plant ht. } \\
(\mathrm{cm})\end{array}$ & $\begin{array}{c}\text { Yield Kg } \\
\text { ha }^{-1}\end{array}$ & $\%$ increase in seed yield over check \\
\hline NFM-8-22 & NM93 x NM92 & 47 & 83 & 46 & 626 & 5 \\
\hline NFM-5-36-1 & VC1560D x NM92 & 46 & 81 & 36 & 765 & 28 \\
\hline NFM-5-36-27 & VC1560D x NM92 & 46 & 82 & 46 & 983 & 65 \\
\hline NFM-5-63-30 & VC1482C x NM92 & 47 & 83 & 40 & 400 & - \\
\hline NFM-5-91-26 & NM98 x VC3902A & 46 & 83 & 40 & 460 & - \\
\hline Ramzan (Check) & VC 1482C x NM 92 & 49 & 83 & 45 & 599 & - \\
\hline $\mathrm{CV}$ & & 0.68 & 2.06 & 3.91 & 7.67 & \\
\hline $\operatorname{LSD}(5 \%)$ & & 0.57 & 4.53 & 2.81 & 90.12 & \\
\hline
\end{tabular}

Table 8. Performance of NFM-5-36-27(NIFA Mung-19) in mungbean adaptation yield trials planted on farmer's field at Kuram Agency during 2016 \& 2017

\begin{tabular}{|c|c|c|c|c|c|c|c|}
\hline \multirow{2}{*}{ Entry } & Parentage & \multirow{2}{*}{$\begin{array}{c}\text { Days to } \\
\text { maturity }\end{array}$} & \multirow{2}{*}{$\begin{array}{c}\text { Plant ht. } \\
(\mathbf{c m})\end{array}$} & \multicolumn{3}{|c|}{ Yield Kg ha-1 } & \multirow{2}{*}{ \% increase in seed yield over check } \\
\cline { 5 - 8 } & & 75 & 66 & 1070 & 2213 & 1642 & \\
\hline NFM-5-36-1 & VC 1560D x NM 92 & 75 & 78 & 1175 & 2534 & 1855 & \\
\hline NFM-5-36-27 & VC 1560D x NM 92 & 78 & 73 & 950 & 2171 & 1561 & \\
\hline NFM-5-91-26 & NM98 x VC 3902A & 78 & 62 & 38 \\
\hline Ramzan (Check) & VC 1482C x NM 92 & 71 & 65 & 812 & 1882 & 1347 & \\
\hline
\end{tabular}


Khattak et al.

Table 9. Mungbean national uniform yield trial conducted by pulses coordinator during Kharif 2015

\begin{tabular}{|c|c|c|c|c|c|c|c|c|c|c|c|c|}
\hline \multirow{2}{*}{ Entry Name } & \multicolumn{11}{|c|}{ Locations (Yield Kg ha ${ }^{-1}$ ) } & \multirow{2}{*}{ Average } \\
\hline & 1 & 2 & 3 & 4 & 5 & 6 & 7 & 8 & 9 & 10 & 11 & \\
\hline 07006 & 817 & 504 & 244 & 111 & 674 & 117 & 490 & 1615 & 1493 & 819 & 709 & 690 \\
\hline 07008 & 740 & 479 & 135 & 73 & 465 & 113 & 420 & 1177 & 1436 & 646 & 819 & 591 \\
\hline NIFA MUNG-4 & 463 & 551 & 106 & 52 & 874 & 114 & 451 & 1625 & 2158 & 764 & 715 & 716 \\
\hline NIFA MUNG-5 & 1072 & 403 & 176 & 42 & 628 & 121 & 309 & 1792 & 2368 & 417 & 653 & 725 \\
\hline NM-14 & 729 & 775 & 106 & 63 & 425 & 135 & 351 & 1854 & 2166 & 694 & 590 & 717 \\
\hline NM-16 & 888 & 428 & 147 & 167 & 683 & 140 & 868 & 2146 & 1711 & 1076 & 875 & 830 \\
\hline NM-17 & 963 & 443 & 154 & 45 & 487 & 154 & 483 & 1500 & 1788 & 903 & 854 & 707 \\
\hline NM-18 & 1272 & 590 & 97 & 97 & 1090 & 125 & 344 & 1885 & 1566 & 882 & 431 & 762 \\
\hline NM-19 & 620 & 628 & 138 & 111 & 822 & 119 & 476 & 1771 & 2173 & 833 & 764 & 769 \\
\hline BRM-356 & 628 & 506 & 150 & 31 & 561 & 131 & 510 & 1240 & 1719 & 938 & 542 & 632 \\
\hline 09-TM-11 & 1079 & 389 & 160 & 125 & 860 & 119 & 580 & 1854 & 1608 & 799 & 681 & 750 \\
\hline 12-TM-03 & 644 & 292 & 197 & 87 & 697 & 121 & 573 & 1563 & 2166 & 674 & 667 & 698 \\
\hline AZ-MH-1 & 719 & 551 & 163 & 153 & 677 & 129 & 455 & 1594 & 1482 & 799 & 653 & 670 \\
\hline AZ-MH-4 & 597 & 418 & 114 & 69 & 578 & 114 & 601 & 2354 & 1310 & 708 & 834 & 700 \\
\hline AZ-MY-6 & 540 & 411 & 110 & 94 & 520 & 114 & 514 & 1646 & 1501 & 924 & 577 & 632 \\
\hline NCM-257-10 & 782 & 107 & 129 & 56 & 217 & 108 & 462 & 1031 & 1161 & 521 & 611 & 471 \\
\hline NCM-252-10 & 831 & 185 & 214 & 56 & 304 & 104 & 684 & 1031 & 1207 & 451 & 514 & 507 \\
\hline AZRI Mung-06 & 749 & 510 & 76 & 118 & 591 & 104 & 493 & 1771 & 1726 & 729 & 833 & 700 \\
\hline NM-2011 & 606 & 469 & 113 & 31 & 580 & 119 & 715 & 1479 & 1413 & 660 & 785 & 634 \\
\hline NM-06 & 655 & 440 & 231 & 90 & 795 & 150 & 476 & 1802 & 1482 & 382 & 632 & 649 \\
\hline
\end{tabular}

\begin{tabular}{|c|c|c|c|}
\hline Locations & Location code & Location \\
\hline $\begin{array}{c}\text { Location } \\
\text { code }\end{array}$ & Location & 7 & BARS, Fateh Jang \\
\hline 1 & NARC, Islamabad & 8 & NIAB, Faisalabad \\
\hline 2 & AARI, Faisalabad & 9 & NIFA, Peshawar \\
\hline 3 & ARI, Mingora,Swat & 10 & RARI, Bahawalpur \\
\hline 4 & ARI, Karak & 11 & BARI, Chakwal \\
\hline 5 & AZRC, D.I.Khan & & \\
\hline 6 & AZRI, Bhakkar & & \\
\hline
\end{tabular}


Table 10. Mungbean national uniform yield trial conducted by pulses coordinator during Kharif 2017

\begin{tabular}{|c|c|c|c|c|c|c|c|c|c|c|c|c|}
\hline \multirow{2}{*}{ S.No } & \multirow{2}{*}{ Entry Name } & \multicolumn{10}{|c|}{ Locations (Yield Kg ha-1) } & \multirow{2}{*}{ Average } \\
\hline & & 1 & 2 & 3 & 4 & 5 & 6 & 7 & 8 & 9 & 10 & \\
\hline 1 & NIFA Mung-4 & 1106 & 917 & 990 & 1314 & 1128 & 535 & 587 & 1265 & 1545 & 801 & 1019 \\
\hline 2 & NIFA MUNG-5 & 928 & 681 & 783 & 735 & 1510 & 490 & 845 & 1138 & 2038 & 809 & 996 \\
\hline 3 & TM-1418 & 1169 & 1104 & 783 & 832 & 1424 & 479 & 573 & 1508 & 1674 & 724 & 1027 \\
\hline 4 & TM-1426 & 1044 & 1083 & 897 & 1157 & 1078 & 465 & 741 & 1643 & 1667 & 749 & 1052 \\
\hline 5 & 13-TM-04 & 1284 & 1271 & 937 & 1094 & 1421 & 462 & 962 & 1252 & 1587 & 785 & 1106 \\
\hline 6 & 13-TM-14 & 1306 & 1021 & 1052 & 900 & 1213 & 431 & 826 & 1494 & 1833 & 850 & 1093 \\
\hline 7 & GV-1 & 1029 & 955 & 810 & 799 & 1778 & 420 & 843 & 1355 & 1531 & 808 & 1033 \\
\hline 8 & MH 3153 & 974 & 1049 & 1100 & 958 & 1108 & 406 & 886 & 1182 & 1670 & 742 & 1008 \\
\hline 9 & MMH 34143 & 854 & 1153 & 879 & 865 & 1178 & 403 & 513 & 1138 & 1170 & 791 & 894 \\
\hline 10 & 120009 & 1032 & 1198 & 849 & 1158 & 2275 & 396 & 729 & 1168 & 1222 & 742 & 1077 \\
\hline 11 & 14002 & 1060 & 708 & 828 & 906 & 1185 & 385 & 515 & 1106 & 1948 & 699 & 934 \\
\hline 12 & MSPS119 & 672 & 833 & 735 & 807 & 1581 & 361 & 1353 & 1348 & 973 & 701 & 936 \\
\hline 13 & NCM-11-2 & 709 & 1063 & 744 & 897 & 1303 & 351 & 1069 & 1037 & 1191 & 752 & 912 \\
\hline 14 & NCM-11-8 & 307 & 944 & 660 & 892 & 1006 & 337 & 602 & 471 & 774 & 772 & 677 \\
\hline 15 & NM-2011 & 1041 & 847 & 982 & 976 & 1136 & 219 & 983 & 1369 & 1413 & 783 & 975 \\
\hline 16 & AZRI Mung-06 & 853 & 646 & 869 & 978 & 1161 & 200 & 928 & 1172 & 1642 & 742 & 919 \\
\hline
\end{tabular}

\section{Locations}

\begin{tabular}{|c|c|c|c|}
\hline $\begin{array}{c}\text { Location } \\
\text { code }\end{array}$ & Location & Location code & Location \\
\hline 1 & AARI, Faisalabad & 6 & BARS, Fateh Jang \\
\hline 2 & ARI, Tando Jam & 7 & NARC, Islamabad \\
\hline 3 & AZRI, Bhakkar & 8 & NIAB, Faisalabad \\
\hline 4 & AZRI, D.I Khan & 9 & NIFA, Peshawar \\
\hline 5 & AZRI, Umer Kot & 10 & ARI, Mingora, Swat \\
\hline
\end{tabular}


In (Table 11) shows Mungbean Yellow Mosaic Virus (MYMV) disease reaction analyses of NFM-5-36-27 (NIFA Mung - 19) along with both parents and standards conducted at NIFA, Peshawar during kharif 2008, 2009 and 2011. Results revealed that NFM-5-36-27 showed resistance to the MYMV disease as compared to one of the parents i.e. VC 1560D which was highly susceptible to MYMV disease with $97.2 \%$ infection. This shows that NIFA Mung -19 possessed more number of favorable modifying genes conferring resistance against MYMV disease as it has been proved that MYMV resistance is the accumulation of such type of genes in a genotype [9]. Furthermore, disease resistance in newly developed crop varieties controls yield losses [18-21], and a new crop variety should surpass the susceptible ones in terms of agronomic performance. The inherent potential of NIFA Mung - 19 for resistance against MYMV disease coupled with high seed yield potential will positively contribute its due share in alleviating mungbean production constraints in KP as well as the country.

Table 11. Reaction of NFM-5-36-27 (NIFA Mung-19) to MYMV as compared to parents and standards during Kharif 2008, 2009 and 2011

\begin{tabular}{|c|c|c|c|c|c|c|c|c|c|}
\hline \multirow{3}{*}{ Entry } & \multicolumn{8}{|c|}{ Mungbean Yellow Mosaic Virus Disease Rating } \\
\cline { 2 - 13 } & At NIFA, Peshawar-2008 & \multicolumn{2}{|c|}{ At NIFA, Peshawar-2009 } & \multicolumn{2}{c|}{ At NIFA, Peshawar-2011 } \\
\cline { 2 - 11 } & $\%$ Infection & $\begin{array}{c}\text { Scor } \\
\mathrm{e}\end{array}$ & $\begin{array}{c}\text { Rati } \\
\mathrm{ng}\end{array}$ & $\begin{array}{c}\% \\
\text { Infection }\end{array}$ & Score & $\begin{array}{c}\text { Ratin } \\
\mathrm{g}\end{array}$ & $\begin{array}{c}\% \\
\text { Infection }\end{array}$ & Score & $\begin{array}{c}\text { Rati } \\
\text { ng }\end{array}$ \\
\hline NFM-8-22 & 7.4 & 2 & $\mathrm{R}$ & 7.1 & 2 & $\mathrm{R}$ & 7.6 & 2 & $\mathrm{R}$ \\
\hline NFM-5-36-1 & 6.9 & 2 & $\mathrm{R}$ & 7.9 & 2 & $\mathrm{R}$ & 7.4 & 2 & $\mathrm{R}$ \\
\hline NFM-5-36-27 & 7.1 & 2 & $\mathrm{R}$ & 7.5 & 2 & $\mathrm{R}$ & 7.7 & 2 & $\mathrm{R}$ \\
\hline NFM-5-63-30 & 4.1 & 1 & $\mathrm{HR}$ & 4.7 & 1 & $\mathrm{HR}$ & 4.1 & 1 & $\mathrm{HR}$ \\
\hline NFM-5-91-26 & 4.4 & 1 & $\mathrm{HR}$ & 4.4 & 1 & $\mathrm{HR}$ & 4.4 & 1 & $\mathrm{HR}$ \\
\hline $\begin{array}{c}\text { NM 92 (Local } \\
\text { parent) }\end{array}$ & 4.2 & 1 & $\mathrm{HR}$ & 4.7 & 1 & $\mathrm{HR}$ & 3.9 & 1 & $\mathrm{HR}$ \\
\hline $\begin{array}{c}\text { VC 1560D } \\
\text { (Exotic parent) }\end{array}$ & 95.8 & 8 & $\mathrm{HS}$ & 97.6 & 8 & $\mathrm{HS}$ & 97.2 & 8 & $\mathrm{HS}$ \\
\hline $\begin{array}{c}\text { Ramzan } \\
\text { (Check) }\end{array}$ & 7.4 & 2 & $\mathrm{R}$ & 7.1 & 2 & $\mathrm{R}$ & 7.3 & 2 & $\mathrm{R}$ \\
\hline
\end{tabular}

MYMV disease score

\begin{tabular}{|c|c|c|}
\hline Plant parts infected/disease (\%) & Score & Disease reaction \\
\hline No infection & 0 & Immune (I) \\
\hline $1-5$ & 1 & Highly resistant (HR) \\
\hline $6-10$ & 2 & Resistant (R) \\
\hline $11-20$ & 3 & Moderately resistant (MR) \\
\hline $21-30$ & 4 & Moderant (T) \\
\hline $31-40$ & 5 & Moderately tolerant (MT) \\
\hline $41-50$ & 6 & Susceptible (S) (MS) \\
\hline $51-80$ & 7 & Highly susceptible (HS) \\
\hline $81-100$ & 8 & \\
\hline
\end{tabular}

NIFA Mung - 19 has an additional future potential for mungbean sprouts production due to its green hypocotyls color which is a pre-requisite for mungbean sprouts production, whereas mungbean varieties adapted to existing growing conditions in Pakistan have purple hypocotyls color. Mungbean sprouts are important part of daily 
diets in many countries e.g. China, Vietnam, Malaysia, Korea, Indonesia etc. [10]. NIFA Mung - 19 has therefore future potential for additional income to the Pakistani growers if proper markets are explored for sprouts consumption in the country.

\section{Conclusion}

NIFA Mung - 19 out-yielded local check variety in all replicated yield trials conducted at NIFA, Peshawar as well as at other locations of the KP, while it produced higher seed yield as compared to the national check varieties in the NUYTs conducted across country for two years. Based on high yield potential, green hypocotyls, disease resistance and different genetic background, KP Provincial Seed Council approved NIFA Mung - 19 as a new mungbean commercial variety for mungbean growing areas of the KP in its $38^{\text {th }}$ meeting held at Agricultural Research Institute (ARI), Peshawar - KP.

\section{Authors' contributions}

Conceived and designed the experiments: GSS Khattak \& I Saeed, Performed the experiments: I Saeed \& M Ibrar, Analyzed the data: I Saeed \& M Ibrar, Contributed materials/ analysis/ tools: GSS Khattak \& I Saeed, Wrote the paper: GSS Khattak \& I Saeed.

\section{Acknowledgement}

Authors of the current manuscript highly acknowledge financial support provided by the Pakistan Science Foundation (PSF) through funded projects "PSF/NSLP/KP/NIFA (128) and PSF/NSLP/KP/NIFA (492)" for carrying out out-station experiments. The authors also highly acknowledge PAEC/NIFA for institutional support to carry-out this research work.

\section{References}

1. Ministry of National Food Security and Research, Islamabad (2017). http://www.amis.pk/Agristatistics/Data/ HTML\%20Final/Moong/Production.ht $\mathrm{ml}$.
2. Hossain M (2012). Rice varietal diversity, milling, and cooking in Bangladesh and Eastern India: A synthesis. In Adoption and diffusion of modern rice varieties in Bangladesh and India, eds, Hossain M, Jaim WMH, Paris TR \& Hardy B, 1-14. Los Banos (Philippines): International Rice Research Institute.

3. Walker TS, Alwang J, Alene A, Ndjuenga J, Labarta R, Yizgezu Y, Diangne A, Andrade R, Andriatsitona RM, De Groote H., Mauch K, Yirga C, Simotowe F, Katungi E, Jogo W, Jaleta M, Pandey S \& Kumara DC (2015). Varietal adoption, outcomes and impact. In Crop improvement, adoption, and impacts of improved varieties in food crops in Sub Saharan Africa, eds, Walker TS \& Alwang J, 388 - 405. Wallingford, UK: CGIAR and CABI.

4. Lantican MA, Braun HJ, Payne TS, Singh R, Sonder K, Baum M, Ginkel MV \& Erenstien O (2016). Impact of international wheat improvement research 1994-2014. Mexico, DF: International Maize and Wheat Improvement Program.

5. Witcombe JR, Khadka K, Puri RR, Khanal NP, Sapkota A \& Joshi KD (2017). Adoption of rice varieties-I. Age of varieties and patterns of variability. Expl Agric 53(4): 512-527.

6. Acharya SS (1969). Comparative efficiency of HYVP-case study of Udaipur District. Econ Polit Wkly 4(44): 1755-1755.

7. Sadiq MS, Saleem M, Haidar S \& Abbas G (2006). NIAB Mung 2006: A high yielding and disease resistant mungbean variety. J Agric Res 44(2): 97-103.

8. Khattak GSS, Ashraf M, Saeed I \& Alam B (2006). A new high yielding mungbean (Vigna radiata (L.) Wilczek) variety "Ramzan". PakJ Bot 38(2): 301310. 
9. Khattak GSS, Haq MA, Ashraf M \& Elahi T (2000). Genetics of mungbean yellow mosaic virus (MYMV) in mungbean (Vigna radiata (L.) Wilczek). J Genet Breed 54: 237-243.

10. Sadikhani MR \& Zeinvand M (2016). Economic evaluation (quantitative) for mungbean (Vignaradiata) production in the Kamin Region, Sadat Shahr, Fars Province. Inter J Med Res Health Sci 5(11): 257-260.

11. Khattak GSS, Haq MA, Rana SA, Elahi $T$ \& Srinives P (1998). An efficient technique for crossing mungbean (Vignaradiata (L.) Wilczek). Thai $J$ Agric Sci 31: 577-582.

12. Steel RGD \& Torrie JH (1980). Principles and procedures of statistics. A Biometrical approach. Mc Graw Hill Book Co., New York (USA).

13. Khan MG, Ahmad W, Khattak G, SirajUd-Din SS \& Ahmad H (2007). Mode of inheritance of resistance to mungbean yellow mosaic virus (MYMV) in mungbean (Vigna radiata (L.) Wilczek). Sarhad J Agric 23(4): 1071-1074.

14. Zulfiqar F \& Hussain A (2014). Forecasting wheat production gaps to assess the future food security in Pakistan. J Food Nutr Disor 3(3): http://dx.doi.org/10.4172/23249323.1000146.
15. Wasim MP (2007). Contribution of High-Yield Varieties Seeds to Major Food Crops Production, Yield and Area in Punjab-Pakistan. Indus J of Manag \& Social Sci 1(1): 46-52.

16. Ahmad M, Akram M, Rauf R \& Khan IA (2007). Adoption and constraints in use of high yielding varieties: A case study of four villages of district Peshawar and Charsada. Sarhad J Agric 23(3): 803806.

17. Joshi KD, Rehman AU, Ullah G, Nazir MF, Zahara M, Akhtar J, Khan M, Baloch A, Elahi E, Khan A, Suleman M \& Imtiaz M (2017). Acceptance and competitiveness new improved wheat varieties smallholder farmers. J of Crop Improv 31(4): 608-627.

18. Agrios G (2005). Plant Pathology. $5^{\text {th }}$ edition, Elsevier, Amsterdam, The Netherlands.

19. Oerke EC, Dehne HW, Schonbeck F \& Weber A (1994). Crop production and crop protection: estimated losses in major food and cash crops. Elsevier, Amsterdam, The Netherlands.

20. Oerke EC (2006). Crop losses to pests. $J$ of Agri Sci 144: 31-43.

21. Savary S, Ficke A, Aubertot JN \& Hollier C (2012). Crop losses due to diseases and their implications for global food production losses and food security. Food Sec 4(4): 519-537. 